\title{
Demographic, Clinical and Therapeutic Effects of Intracranial Meningioma
}

\author{
Alireza Khoshnevisan ${ }^{1 *}$, Seyyed Mohsen Hasani Barzi ${ }^{2}$, Sepideh Shahi ${ }^{3}$ and Fansu FN Jatta ${ }^{4}$ \\ ${ }^{1}$ Neurosurgeon and Faculty Member of Tehran University of Medical Sciences, Iran \\ ${ }^{2}$ Neurosurgeon and Faculty Member of Qazvin University of Medical Sciences, Iran \\ ${ }^{3}$ Medical student, Tehran University of Medical Sciences, Iran
}

${ }^{4}$ Resident of Neurosurgery, Tehran University of Medical Sciences, Iran

*Corresponding author: Alireza Khoshnevisan, Neurosurgeon and Faculty Member of Tehran University of Medical Sciences, Iran

\section{ARTICLE INFO}

Received: 幽 November 11, 2019

Published: 幽 November 19, 2019

Citation: Alireza Khoshnevisan, Seyyed Mohsen Hasani Barzi, Sepideh Shahi, Fansu FN Jatta. Demographic, Clinical and Therapeutic Effects of Intracranial Meningioma. Biomed J Sci \& Tech Res 23(1)-2019. BJSTR. MS.ID.003839.

Keywords: Meningioma; Demographic effects; Clinical presentation; Therapeutic outcome

Abbreviations: CPA: Cerebello-Pontine Angle; CSF: Cerebrospinal Fluid; GTR: Gross Total Resection; HIS: Health Information System; SD: Standard Deviation; SPSS: Statistical Package for Social Sciences; WHO: World Health Organization

\begin{abstract}
Purpose of the Study: Meningioma is one of the most common intracranial tumors originating from the meninges. Due to its high prevalence, having sufficient and accurate information about the epidemiology, patient characteristics, and surgical outcomes is of great clinical significance. The aim of this study is to determine the demographic, clinical, and therapeutic outcome of intracranial meningiomas in patients undergone surgery in Tehran University of Medical Sciences during 2009-2014.
\end{abstract}

Materials and Methods: This study was performed by cross-sectional study on patients' files. Ninety two (92) files of patients diagnosed with meningioma formed the samples from the three major educational hospitals of Tehran University of Medical Sciences. Data gathering process in this study includes demographic information of patients, tumor characteristics, and treatment outcomes. Data were primarily collected from patients' files. In some cases, information was obtained by telephone calls with patients and telephone interviews.

Results: The mean age of the patients was $52.87 \%$, and the majority of the samples were female (74\%). 58.7\% of patients referred to the hospital with headache. The most frequent tumor site was parasagittal and falx and $54.3 \%$ of brain tumors were adjacent to the brainstem. $85.9 \%$ had first-degree tumors, and in $51.1 \%$ had a volume between 11- $40 \mathrm{ml}$ cubic meters. In $35.9 \%$ of cases, there was edema around the tumor. In $78.3 \%$ of cases, gross total resection (GTR) was achieved at surgery. The mortality rate and postoperative recurrence were $4.4 \%$ and $16.3 \%$, respectively. There was a significant relationship between tumor volume and edema around the tumor.

Conclusion: The study revealed the relationship between epidemiological factors, clinical presentations, and therapeutic surgical outcomes regarding the management of meningioma.

Most of the meningiomas were observed to present in the elderly women, and headache was found to be the main presenting complaint. Gross total resection (GTR) was the major modality of treatment and has significant favorable outcome. More research especially on risk factors, conditions predisposing to meningioma reoccurrence, and primary symptoms and signs of the disease, would go a long way to providing better diagnosis and improved treatment outcomes.

\section{Introduction}

Meningioma is one of the most common brain tumors. The overall prevalence of meningioma is 6.59 per 100,000 population.
In a study conducted using general cerebral MRI in the general population, the prevalence of meningioma was reported to be $0.9 \%$ 
$[1,2]$. The prevalence of this disease has been reported differently in different populations, with a white Hispanic population of 6.53 per 100,000 population, African Americans, 29.7 per 100,000 population, and in the non-Hispanic white population was 6.38 per 100,000 [3]. Meningioma originates from arachnoid cap cells and is often benign and grows slowly. The most common place of occurrence of meningioma is at the base of the skull. Other places in the brain that meningioma can be seen include the optic region and meningioma inside intraventricular or choroid plexus [2]. Meningioma can rarely be extra- cranial, which is most commonly found in areas such as the middle ear, temporal bone, sino-nasal cavity, orbit, oral cavity, parotid gland, in soft tissues of the neck [4] . Meningiomas in spinal cord also occur in $80 \%$ of cases in the thoracic region [2].

Although the main cause of meningioma is unknown, but factors such as age, head and neck radiotherapy, genetic conditions and familial history, head trauma, chronic viral infection and cigarette smoking have all been implicated in the aetiology. Occupation, diet regimen, and history of allergies are also cases that have been proposed to have effect on the incidence of meningioma [1,3,5]. Studies revealed the role of genetic factors as limited, but hereditary genetic syndromes such as neurofibromatosis have been observed to contribute in the aetiology of meningioma [3].

Meningioma can be classified according to the main location of the dura and surrounding tissue involvement (e.g., venous sinus, bone, brain, and nerves) and according to World Health Organization (WHO) criteria of tumor grading [2]. According to the classification of the WHO, grade I is a benign meningioma, grade II is categorized as atypical meningioma and grade 3 is classified as anaplastic. WHO further categorized that $92 \%$ of meningiomas are benign and are of grade 1 type, 6\% are atypical and Grade II, and $2 \%$ were reported to be malignant $[1,6]$. Some studies also categorized meningiomas into two molecular groups, one category all invasive meningiomas ( such as recurrent meningiomas), and the other group, are the benign and atypical meningiomas [3]. The radiological findings of the lesion are usually non-specific, but the presence of a typical dural tail after contrast enhancement is more characteristic. Macroscopically, the tumor is a rigid gray mass of white to pink that has a gravel consistency when sliced [4].

Various therapies for meningioma include surgery, stereotactic surgery, hormone therapy, chemotherapy or targeted therapy. Hormone therapy, chemotherapy, or targeted therapy are mainly employed in the treatment of recurrent meningiomas [3]. The survival rate after 5 years of diagnosis of meningioma is reported to be $64 \%$, of which $70 \%$ were reported in women and $55 \%$ in men. According to reports, 170,000 men with meningioma are living in the United States currently [7]. The prevalence of central nervous system tumors, including meningioma, has been reported in different societies based on age, gender, anatomical location and different pathology, and it is important to examine and report on various epidemiological and clinical aspects, since diagnosis in the early stages with radiographic methods are possible [8]. Also, due to the prevalence of this disease in the active age group of society (fourth and fifth decades of life), timely diagnosis and identification of etiological factors can lead to effective treatment and the resultant improvement in health of the individuals with reduction in mortality and morbidity [9] .

Several studies have examined the incidence of intracerebral meningioma. But few studies have been conducted on the epidemiology and etiology of this disease [1], and in Iran there is limited research done. It should also be noted that studies that have been carried out to determine the prevalence of this disease were in the past [8,9] or in specific populations [10], or in brain tumors in general [11]. Thus, the study of the demographic characteristics, clinical and therapeutic implications of intracranial meningioma becomes critical and important. This research work is not exclusive to a specific population or region but can be examined in each country to identify and prevent the risk factors that would help researchers to apply solutions to reducing the high prevalence. Studies have shown that in the developed countries there are programs for studying the prevalence and aetiology of meningioma. In Iran, there are limited epidemiological studies on meningioma, and considering its high prevalence and economic importance more research cannot be overemphasized. Therefore, the aim of this study was to determine the demographic characteristics, clinical, and therapeutic outcome of intracranial meningiomas in Tehran hospitals affiliated to Tehran University of Medical Sciences from 2009 to 2014.

\section{Materials and Methods}

This study was a cross-sectional study in which the demographic characteristics, clinical and therapeutic effects of intracranial meningioma were examined and described in Imam Khomeini Hospital, Shariati Hospital and Sina Hospital affiliated to Tehran University of Medical Sciences. The patients in the study were men and women over the age of 18 years, with any occupation, level of education and marital status, all diagnosed with intracranial meningioma who underwent surgery in Imam Khomeini Hospital, Shariati Hospital and Sina Hospital affiliated to Tehran University of Medical Sciences. The study sample comprised of 92 patients who were hospitalized in the various hospitals between 2009 to 2014. Considering the fact that the above three centers were the main centers of neurosurgical operations in Iran, therefore a quantitative sampling was performed such that from each hospital, at least 30 case files of patients were examined. The records were obtained from surgical offices in Neurosurgery in conjunction with the HIS system of the hospital. A random table number was used for sampling. In this way each hospital, in order to reach the minimum number of 30 case files, the statistics of patients were sorted according to the year (between 2009 and 2014) and according to the random table numbers, patients were selected from this list. This method continued until the number of samples were obtained. After specifying the samples and extracting the file numbers of these patients from the HIS system of the hospital archives, the file of the patients was studied. 
The data gathering tool in this study was prepared after reviewing the relevant texts, combining and integrating with the integral neurosurgical tools, as well as opinion of the relevant experts by the researcher. For determining the scientific validity, a content validity was used to gather data considering the research goal, reference books, collected articles related to the subject, publications and scientific researches. Then, the validity of this tool was assessed by five members of the Faculty of Neurosurgical Assessment and Corrective Committee. A case report such as relapse disease, death, re-operation, depression and anxiety was done by phone call to patients. All information needed by the researcher were collected and thus the bias of researchers in data collection was reduced. Data were entered into SPSS 12 software and were analyzed using descriptive and analytical statistics.

\section{Research Results}

The total number of samples in this study was 92 cases of patients who had undergone surgery due to intracranial meningioma. Table 1 shows the frequency of the studied variables. This table shows that the age range of patients in this study was between 23 - 88 years and the mean and standard deviation $(63.12$ years) were 52.78 years (SD). The majority (74\%) of the studied units were women and the rest were men with a prevalence of $26 \%$. In most cases (78.2\%), headache was part of the complaint that patients referred to the hospital. The highest frequency of tumor site $(21.7 \%)$ was related to para-sagittal and falx and the next frequency was related to convexity(17.4\%) and wing of sphenoid (15.2\%) respectively. The highest frequency of tumor grade was related to benign tumor Grade I (85.9\%). In 54.3\% of the cases, the tumors were proximal to the brainstem. The majority of tumors (51.1\%) had a size between $11-40 \mathrm{ml}$ cubic meters. In $35.9 \%$ of cases, edema around the tumor existed. In $78.3 \%$ of the cases, the GTR method was used to resect the tumor and in $70.7 \%$ of the surgeries the microscope was employed. In $4.4 \%$ of cases, patients died after surgery and the rate of recurrence after surgery was $16.3 \%$. The mean time of surgery until recurrence was 23.17 months with a standard deviation of 12.37 months. $2.2 \%$ of the patients had a history of radiotherapy, $2.2 \%$ had a history of head traumas, $7.6 \%$ had history of diabetes, $21.7 \%$ had a history of high blood pressure and $8.7 \%$ had a history of cardiovascular disease. $10.9 \%$ of patients had hematoma after surgery. Few of them were infected $(2.2 \%)$. In $31.5 \%$ of patients, CSF leaks and hydrocephalus were observed. Few patients (4.3\%) had neurological complications and $22.8 \%$ of patients had depression and $8.7 \%$ had anxiety Table 2 .

Table 1: Absolute Frequency and Percentage of Target Units on the Background Variables.

\begin{tabular}{|c|c|}
\hline variation & Frequency/ (percentage) (standard mean deviation) \\
\hline age & $52 / 78(12 / 63) *$ \\
\hline \multicolumn{2}{|l|}{ sex } \\
\hline female & $(74) 68$ \\
\hline male & $(26) 24$ \\
\hline \multicolumn{2}{|l|}{ Primary compliant } \\
\hline Only headache or with other symptoms & $72(78 / 2)$ \\
\hline Auditory loss & $2(2 / 2)$ \\
\hline Hemaplasia and weakness & $4(4 / 3)$ \\
\hline seizures & $10(10 / 9)$ \\
\hline Light headedness & $4(4 / 3)$ \\
\hline \multicolumn{2}{|l|}{ Tumor location } \\
\hline para saggittal and falci & $20(21 / 7)$ \\
\hline Convexity & $16(17 / 4)$ \\
\hline Sphenoid Wing & $14(15 / 2)$ \\
\hline Olfactory Groove & $8(8 / 7)$ \\
\hline $\mathrm{CPA}$ & $11(12)$ \\
\hline Others (sinus carvanosus foramen magnum intra-ventricle & $25(27 / 2)$ \\
\hline \multicolumn{2}{|l|}{ Tumor grades } \\
\hline Grade I (benign) & $(9 / 85) 79$ \\
\hline (atypical meningioma) Grade II & $(7 / 8) 8$ \\
\hline Grade III (aplastic meningioma) & $(4 / 5) 5$ \\
\hline \multicolumn{2}{|l|}{ Tumor migration to brainstem } \\
\hline Present & $(3 / 54) 50$ \\
\hline Absent & $(7 / 45) 42$ \\
\hline \multicolumn{2}{|l|}{ Tumor size } \\
\hline $10 \geq$ & $(7 / 33) 31$ \\
\hline
\end{tabular}




\begin{tabular}{|c|c|}
\hline $40-11$ & $(1 / 51) 47$ \\
\hline $41 \leq$ & $(2 / 15) 14$ \\
\hline \multicolumn{2}{|l|}{ Edema around tumor } \\
\hline Absent & $(1 / 64) 59$ \\
\hline Present & $(9 / 35) 33$ \\
\hline \multicolumn{2}{|l|}{ Type of surgery } \\
\hline GTR & $72(78 / 3)$ \\
\hline near total & $5(5 / 4)$ \\
\hline Partial & $13(14 / 1)$ \\
\hline \multicolumn{2}{|l|}{ Using microscope for surgery } \\
\hline Yes & $(7 / 70) 65$ \\
\hline No & $(3 / 29) 27$ \\
\hline \multicolumn{2}{|l|}{ Survival after surgery } \\
\hline Alive & $(5 / 93) 86$ \\
\hline Dead & $(4 / 4) 4$ \\
\hline \multicolumn{2}{|l|}{ Relapse after surgery } \\
\hline No & $(7 / 83) 72$ \\
\hline Relapse & $(3 / 16) 14$ \\
\hline Duration between surgery and relapse & $23 / 7(12 / 37)^{*}$ \\
\hline \multicolumn{2}{|l|}{ Underlying disease } \\
\hline History of radiotherapy of head & $2(2 / 2)$ \\
\hline Head trauma & $2(2 / 2)$ \\
\hline Diabetes & $7(7 / 6)$ \\
\hline Hypertension & $20(21 / 7)$ \\
\hline Cardiovascular disease & $10(10 / 9)$ \\
\hline \multicolumn{2}{|l|}{ Complication after surgery } \\
\hline Hematoma after surgery & $10(10 / 9)$ \\
\hline Infection /sepsis & $2(2 / 2)$ \\
\hline CSF leak and hydrocephalus & $29(31 / 5)$ \\
\hline Neurologic complication & $4(4 / 3)$ \\
\hline Depression & $21(22 / 8)$ \\
\hline anxiety & $8(8 / 7)$ \\
\hline
\end{tabular}

Table values are based on the frequency (percentages) except in starred cases * marked with an average (SD

Table 2: Frequency of tumor recurrence rate based on tumor location.

\begin{tabular}{|c|c|c|c|}
\hline Tumor location & Number of meningioma resected & Number of relapse (percentage) & percentage of relapse in every \\
\hline Cavernous Sinus & 5 & $1(20)$ & $1 / 7$ \\
\hline Convexity & 16 & $2(12 / 5)$ & $14 / 3$ \\
\hline CPA & 11 & 0 & 0 \\
\hline Foramen Magnum & 2 & 0 & 0 \\
\hline Intra ventricular & 1 & 0 & 0 \\
\hline Olfactory Groove & 8 & $2(25)$ & $14 / 3$ \\
\hline Para saggittal and falci & 20 & $1(5)$ & $7 / 1$ \\
\hline Petroclival & 3 & 0 & 0 \\
\hline Sphenoid Wing & 14 & $(8 / 42) 6$ & $42 / 8$ \\
\hline Tentorial & 6 & $(6 / 16) 1$ & $7 / 1$ \\
\hline Tuberculum Sellae & 6 & $(6 / 16) 1$ & $7 / 1$ \\
\hline Summation & 92 & $(100) 14$ & 100 \\
\hline
\end{tabular}


There was a significant relationship between peritumoural oedema and tumor size. In $26.1 \%$ of cases that had tumor size between 11- $40 \mathrm{ml}$ cubic, there was pertumoural oedema, whilst of the 14 cases of tumor size greater than $40 \mathrm{ml}$ cubic meters, there were $8 \%$ cases of edema around the tumor. In other words, with increased tumor size, the peritumoral oedema also increased
Table 3. The results further revealed that there was no statistically significant relationship between age and recurrence of the disease, tumor grade and postoperative relapse, type of surgery and postoperative recurrence, edema around the tumor and recurrence after surgery. Also, there was no significant relationship between relapse rate and type of surgery in terms of tumor grade Table 4.

Table 3: Relationship between tumor edema and tumor size in research units.

\begin{tabular}{|c|c|c|c|c|}
\hline & Edema around tumor number & & & \\
\hline Tumor size & \multicolumn{2}{|c|}{ (percentage) } & Test type & $p$-value \\
\hline & Yes & No & \multirow{4}{*}{$X^{2}=21 / 832$} & \multirow{4}{*}{$0 / 000$} \\
\hline $10 \geq$ & $(1 / 1) 1$ & $30(32 / 6)$ & & \\
\hline $10 \geq$ & $24(26 / 1)$ & $23(25 / 0)$ & & \\
\hline $40 \leq$ & $8(8 / 7)$ & $6(6 / 5)$ & & \\
\hline
\end{tabular}

Table 4: Relationship between tumor recurrence and other variables in the studied units..

\begin{tabular}{|c|c|c|c|c|c|}
\hline & & Recurrence & & & \\
\hline & & (Yes) $\mathrm{N}=14$ & (No) $\mathrm{N}=72$ & Test type & $p$-value \\
\hline & Age & $50 / 7(10 / 12$ & $52 / 8(12 / 60)$ & $\mathrm{T}$ Test $=0 / 604$ & $0 / 334$ \\
\hline \multicolumn{6}{|c|}{ Tumor Grade } \\
\hline & Grade I & $(0 / 14) 12$ & $(4 / 74) 64$ & & \\
\hline & Grade II and III & $(3 / 2) 2$ & $(3 / 9) 8$ & $F=0 / 663$ & $512 / 0$ \\
\hline \multicolumn{6}{|c|}{ Type of surgery } \\
\hline & GTR & $(1 / 13) 11$ & $(9 / 67) 57$ & & \\
\hline & Near total \& Partial & $(6 / 3) 3$ & $(5 / 15) 13$ & $X^{2}=0 / 062$ & $804 / 0$ \\
\hline \multicolumn{6}{|c|}{ Edema around tumor } \\
\hline & Yes & 6 & 24 & $X^{2}=0 / 468$ & $494 / 0$ \\
\hline & No & 8 & 48 & & \\
\hline \multicolumn{6}{|c|}{ Grade and surgery type } \\
\hline \multirow[t]{3}{*}{ Grade I } & GTR & 10 & 50 & & \\
\hline & Near Total & 0 & 5 & $X^{2}=0 / 340$ & $0 / 324$ \\
\hline & partial & 2 & 7 & & \\
\hline \multirow[t]{2}{*}{ Grade II } & GTR & 1 & 4 & $X^{2}=0 / 343$ & $0 / 587$ \\
\hline & Partial & 1 & 1 & & \\
\hline
\end{tabular}

\section{Discussion}

This study was an insight into meningioma and its demographic, clinical and therapeutic implications in meningeal patients referred to the three major centers of neurosurgery. The results of this study were consistent with various other researches. For example, the results showed that the average age of the patients was 52.87 years. The chance of developing meningioma increases with age, and this is true of both male and female sex. In addition to the higher prevalence of meningioma with age, the highest incidence occurs after 50 years of age. In the study in England, the mean age of diagnosing meningioma was 62.62 years in women and 62.2 years old in men [2]. In the study conducted in Iran and in Rasht, the average age of 62 patients who had undergone surgery due to meningioma was reported to be 49.1 [8]. In the study in Kerman, the mean age of patients with meningioma was 52.2 years and peak age of these tumors was 40 - 70 years [12]. The results of these studies are consistent with the results of the index study. In addition, the majority of samples (74\%) were female, and this ratio was 2.6 to 1. This means that the incidence of meningioma in women was 2.6 times higher. These results are consistent with the results of studies conducted in this area. Meningioma is more common in women than men in numerous studies $[2,13,14]$, and the ratio of meningeal outbreaks in women is 2.6 to 1 [2]. In a study in Iran and in Rasht in meningioma patients, the ratio of female to male is 1.6 to 1 (8). Moreover, in the study in Kerman, it was 2.4 to 1 [12].

Regarding the high incidence of meningioma in women, numerous studies have shown that there is a significant relationship between hormones and the risk of meningioma [3]. This could be 
due to the presence of estrogen receptors on meningioma cells in women explaining the increase in meningioma size during pregnancy and menstrual cycles [8]. Out of 92 patients, 54 patients (58.7\%) referred with a headache complaint or had a headache with loss of vision (14.1\%) or imbalance (4.5\%) [15]. In research studies, headache is one of the most important meningioma symptoms mentioned [1]. Others have pointed out that although the clinical symptoms of meningioma vary according to the location and size of the tumor, they are often asymptomatic and have slow growth; but when symptoms occur, headache and headache associated with seizure are the most important presentation [16]. In some studies, a range of symptoms has been reported from headache, seizure and organ weakness depending on the location of the anatomical tumor [17]. In studies done in Iran and in Rasht by Hatamy, it was also reported that headache was the most common symptom and presented in $85.5 \%$ of patients with meningioma [8].

In addition, the results showed that the most frequent tumor sites were para-sagittal and falcine, followed by tumors of the convexity and sphenoid wing. These results are consistent with the results of other studies. The most common types of meningioma have been reported based on the anatomical location of the convexity brain (35\%), parasagittal (20\%), and sphenoid (20\%) and intra-ventricular (5\%) (17). In the study in Rasht, the most frequent site of involvement was the brain convexity (37.1\%), parasagittal sinus (24.2\%), and sphenoid wing (17.7\%) (8). In another study based on the highest frequency of meningioma, it was the sinus region of cavernous and sella turcica (45\%), parasagittal area (22\%), and cerebellopontine angle (14\%) [18]. In the present study, the results further showed that more than half of the tumors in the samples (54.3\%) had a proximity to the brain stem. One of the reasons for this finding is that a tumor lying in proximity with the brain stem is dangerous and needs advanced equipment, and since Imam Khomeini Hospital, Shariati and Sina are referral centers of neurosurgery in Iran, and are better equipped in terms of surgical and medical techniques, patients with high risk of surgery were referred to these centers.

The results of the index study showed that first-degree benign tumors were the most abundant (85.9\%), Grade II tumors, or atypical meningioma (8.7\%), and Grade III, or anaplastic meningioma $(4.5 \%) \%$ ). These results are consistent with other studies. For example, in a study conducted in Iran in 2013, 89 of the meningiomas examined were $43.22 \%$ of the grade $1,44.94 \%$ of the grade $2,37.3 \%$ of the grade three. $49.4 \%$ were meningioma with atypical appearance and $3.37 \%$ were invasive brain meningioma [19]. The World Health Organization (WHO) has also pointed out that grade I meningiomas are the most common but associated with relatively low risk of relapse, while those with higher degrees are associated with increased risk of recurrence and aggressive behavior [20]. The results further revealed that in half of the cases (51.1\%) tumors had a size between $11-40 \mathrm{ml}$ cubic meters. A study in Shiraz within a 20-year period showed that 382 tumors had a small size and 262 tumors of a large size (9). In a study by Bitaraf and associates specifically in radiosurgery in meningioma, by the volume of tumor in milliliters (tumor volume range) had a range between 7 and 30 [18], which is somewhat consistent with the results of the present study.

The results of this study also showed that in more than half (64\%) of patients the peritumoral edema was not present, but in other cases edema (35.9\%) was observed. In a study conducted in Iran by Bitaraf and colleagues under the title "Peri-tumor edema associated with meningioma," in $54.6 \%$ of the patients, there was a peri-tumoral edema, of which the largest diameter of the edema was $1.6 \mathrm{~cm}$, and in 27 patients with localized edema there were 15 diffused edema [21]. In a study in Rasht, tumor edema was observed in $59.7 \%$ of cases (8). One of the reasons for the difference in the results of the studies mentioned and this recent study can be the measurement of edema around the tumor and its diagnostic criteria. The majority of surgical procedures performed $(78.3 \%)$ were done by GTR, followed by subtotal resection (14.1\%). The sources point out that the closer these tumors are to vital elements of the brain, causes complications of the microscopic surgery of these tumors [18].

In addition, since the treatment centers in which this research was conducted is a national referral center, and since the results of this study showed that most of the tumors (54.3\%) were adjacent to the brain stem, GTR surgery for these patients were more safer. In addition, this results showed that $51.1 \%$ of tumors had a size between 11 to $40 \mathrm{ml}$ cubic meters and $15.2 \%$ of them had a size greater than $41 \mathrm{ml}$ cubic meters which is a justification of the GTR surgical technique. Again, the location of the tumor can also affect the type of surgery. As the results of this study showed, $21.7 \%$ of tumors were in Falcine and Parasagittal, 17.4\% in convexity, 15.2\% in sphenoid wing, which is a risky site for surgery. In addition, $12 \%$ of these tumors are located on the cerebellopontine angle. The results of this study showed that $16.3 \%$ of cases had post-operative recurrence. In a study done in 20-year period by Farkhi and colleagues, 62 of 644 patients with cerebral meningioma suffered relapse, the rate of recurrence reported to be $6.9 \%$.

The chance of recurrence was higher in tumors with peritumoural oedema and bone changes, and the chance of recurrence of the tumor was reduced by increasing the degree of surgical resection. Malignant and atypical types of meningiomas have higher rates of relapse than benign types, in other words, with increased in tumor grade the chance of recurrence increases [9]. In other studies, it was specified that the probability of recurrence in intracranial meningioma increased by $20 \%$ for each $1 \mathrm{~cm}$ of edema [21]. In various studies, the most important effective factor in predicting the rate of recurrence is the degree of surgical resection of the tumor (the higher the degree of resection, the lower the tumor recurrence). In a study conducted in Japan, the recurrence rate was $9 \%$ in grade1, in the grade $216 \%$, and $29 \%$ in the grade 3 [22]. 
Concerning complications after surgery, the results showed that $31.5 \%$ of patients had hydrocephalus and CSF leak, $10.9 \%$ had hematoma, infection (2.2\%) and neurological sequelae (4.3\%). The study further observed that $22.8 \%$ of patients developed depression and $8.7 \%$ anxiety. Anxiety and depression in these patients can be due to the nature of the illness, physical complications caused by the operation, financial issues following the functional decline of these patients and the fear of the future [23]. The results of a study conducted in Iran on quality of life and its related factors in patients with brain tumors showed that these patients had low quality of life in all aspects of the general and specific. They had the lowest scores in the overall quality of life and emotional functioning and had very low assurance for the future [24]. In terms of survival, the study revealed that $93.5 \%$ survived post operation with $4.4 \%$ passed-away from surgery up to 30 days or beyond. Death from meningioma surgery occur mainly from brain damage, or vascular events (damage to carotid or brainstem arteries) [18]. In a five-year study, the survival rate was $78 \%$ in Meningioma Grade II patients and $44 \%$ in Grade III and malignant meningioma [25]. In the study of Firouzian, on the study of the relative frequency of meningioma in patients referred to the department of Radiotherapy and Oncology in Ahvaz, Golestan Hospital during the years 2001 to 2012 , the mortality rate was reported to be $10.7 \%$ [15]. In this study, the mortality rate was lower than other studies, which could be attributed to advanced surgical techniques, surgeon factors and patients' factors.

The results of this study also showed that there was significant relationship between size of the tumor and peritumoural oedema, in the respect that with greater tumor size, edema increases. This phenomenon can be explained by the theory of "Hydrodynamic expansion from the tumor to the normal parenchyma". It is postulated that with the loss of the buffer layer between the tumor and the normal brain parenchyma, a diffusion gradient is created that allows the transfer of macromolecules leading to oedema around the tumor periphery [21].

\section{Study Limitations}

Data were collected retrospectively, and some information from meningioma data collection process were not mentioned or not available in in-patient records. However, the researchers tried to carefully review the patient records for adequate relevant information. The occurrence of some disorders such as depression and anxiety caused by intracranial meningioma requires collecting specific data which was not possible due to limited access to patients and the retrospective nature of the study, leading to such information being collected by self-declaration of patients.

\section{Conclusion and Recommendations}

The study revealed the relationship between the epidemiological factors, clinical consideration, and therapeutic surgical outcome as regards the management of meningioma. Most of the meningiomas were observed to be present in the elderly women with headache as the main complaint at presentation. GTR being the major treatment modality has significant favorable outcome. Hence, understanding the epidemiological events together with the tumor clinicopathological characteristics is important for better planning. Thus, more research especially on the risk factors, conditions predisposing to reoccurrence, and precise clinical presentations would go a long way to improving care and quality of life of the patient leading to reduction in morbidity and mortality.

\section{Declarations}

\section{Ethics Approval and Consent to Participate}

In order to proceed with the research, approval of the respected Research Affiliation of the Tehran University of Medical Sciences and the Head of the mentioned hospitals were obtained. All patient information's were kept confidential and each patient was assigned a secret code.

\section{Consent for Publication}

Informed consent for publication of this manuscript was obtained.

\section{Availability of Data and Material}

Please contact author for data request.

\section{Competing Interest}

The authors declare that there is no competing interest in the publication of this manuscript.

\section{Funding}

The authors declare no source of funding for this research.

\section{Authors' Contributions}

Not applicable.

\section{Acknowledgement}

Not applicable.

\section{References}

1. Cea Soriano L, Wallander MA, Garcia Rodriguez LA (2012) Epidemiology of meningioma in the United Kingdom. Neuroepidemiology 39(1): 2734.

2. Alexiou GA, Gogou P, Markoula S, Kyritsis AP (2010) Management of meningiomas. Clinical Neurology and Neurosurgery 112(3): 177-182.

3. Chamberlain MC, Barnholtz Sloan JS (2011) Medical treatment of recurrent meningiomas. Expert review of neurotherapeutics 11(10): 1425-1432.

4. Hosseinpour P, Hashemi F (2006) Extracranial Meningioma: A Case Report. Razi Journal of Medical Sciences 13(50): 47-50.

5. Wiemels J, Wrensch M, Claus EB (2010) Epidemiology and etiology of meningioma. Journal of neuro oncology 99(3): 307-314.

6. Kleihues P, Louis D, Scheithauer B, Rorke L, Reifenberger G, et al. (2002) The WHO classification of tumors of the nervous system. Journal of Neuropathology \& Experimental Neurology 61(3): 215-225.

7. Simó M, Izquierdo C, Bruna J (2013) Systemic treatment of recurrent meningioma. European Association of NeuroOncology Magazine 3(3): 132-138. 
8. Hatamian H, Sobhani A, Emam hadi M (2004) A Six- Year Review of Meningioma in Rasht Poorsina Hospital. Journal of Guilan University of Medical Sciences13(49): 39-45.

9. Farrokhi M, Ansari Z (2006) Recurrence of intracranial meningioma and its contributing factors: a 20 -year study. Tehran University Medical Journal TUMS Publications 64(12): 91-96.

10. Taghipour M, Razmkon A, Bakhtazad A, Mousa, Atefeh (2010) High Prevalence of Intracranial Meningioma in Jewish Population in Shiraz Southern Iran. Neurosurgery Quarterly 20(2): 68-70.

11. Jazayeri SB, Shokraneh F, Ramezani R, Alimi M, Saadat S, et al. (2013) Epidemiology of Primary Brain and Spinal Cord Tumors in Iran: a systematic review. The Neuroscience Journal of Shefaye Khatam 1(2): 13-20.

12. Hamed Rek (2004) Epidemiologic study of brain tumors in Kerman during 1997-1998. Two Journal of South medicine(1): 47-53.

13. Jazayeri SB, Rahimi Movaghar V, Shokraneh F, Saadat S, Ramezani R (2013) Epidemiology of Primary CNS Tumors in Iran: A Systematic. Asian Pacific Journal of Cancer Prevention 14(6): 3979-3985.

14. Amir al (2003) Surveying the prevalence of age and sex in one hundred cases of meningioma at Imam Hossein Medical Center, Tehran. Shahid Beheshti University of Medical Sciences. Shahid Beheshti University of Medical Sciences.

15. Rosita F (2014) study of Relative frequency of Meningioma in Patients Referring to the Radiation Therapy and Oncology Department of Ahvaz Golestan Hospital from 2001 to 2012. Ahvaz: Jondishapour University of Medical Sciences and Health Services, Ahwaz, Iran.

16. Marta GN, Correa SFM, Teixeira MJ (2011) Meningioma: review of the literature with emphasis on the approach to radiotherapy. Expert Review of Anticancer Therapy 11(11): 1749-1758.

\section{ISSN: 2574-1241}

DOI: 10.26717/BJSTR.2019.23.003839

Alireza Khoshnevisan. Biomed J Sci \& Tech Res

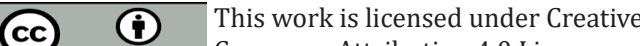
Commons Attribution 4.0 License

Submission Link: https://biomedres.us/submit-manuscript.php
17. Haddadi K, Asadian L, Khademloo A (2012) Parasagittal Meningioma: A mimic of Cerebrovascular Accident. Journal of Mazandaran University of Medical Sciences 22(95): 105-109.

18. Tayebi Meybodi A, Sharif Tabrizi A, Alikhani M, Bitaraf MA, Azar M, et al. (2010) Radiosurgery for skull base meningiomas: a study on 230 cases in Iranian Gamma Knife Center. Tehran University Medical Journal TUMS Publications 68(3): 162-167.

19. Khuzani Sh, Moradi, Oneani P, Farid S, Majd (2013) Pathological examination of meningioma with ki 67 and p53 markers in patients referred to Shohada-e-Tajrish Hospital. Research in Medicine 37(2): 102-106.

20. Ali Beygzadeh (2015) Meningioma: Features and Treatment of Surgery. Monthly Nada Miaha (61):36-2.

21. B Alikhani, Mazdak, Saberi, Hashemi, Sedighi, et al. (2004) Peri-tumor edema associated with meningioma, role of steroid receptors and radiological specifications. Found 6(1): 3-11.

22. Yamasaki F, Yoshioka H, Hama S, Sugiyama K, Arita K, et al. (2000) Recurrence of meningiomas. American Cancer Society 89(5): 11021110 .

23. Mehri S, Davar A, Mahast N (2016) Primary manifestation of brain tumors in patient with brain tumor referred to neurology clinical 2016.

24. Pakpour Hajibaba A, Yekaninejad MS, Khoshnevisan AR, Mikbakht M, Mardani A, et al. (2013) Assessement of The Quality of Life and Its Related Factors Among Patients with Brain Tumors. scientific Journal of Ilam University of Medical Sciences Scie 21(3).

25. Durand A, Labrousse F, Jouvet A, Bauchet L, Kalamaridès M, et al. (2009) WHO grade II and III meningiomas: A study of prognostic factors. Journal of neuro-oncology 95(3): 367-375

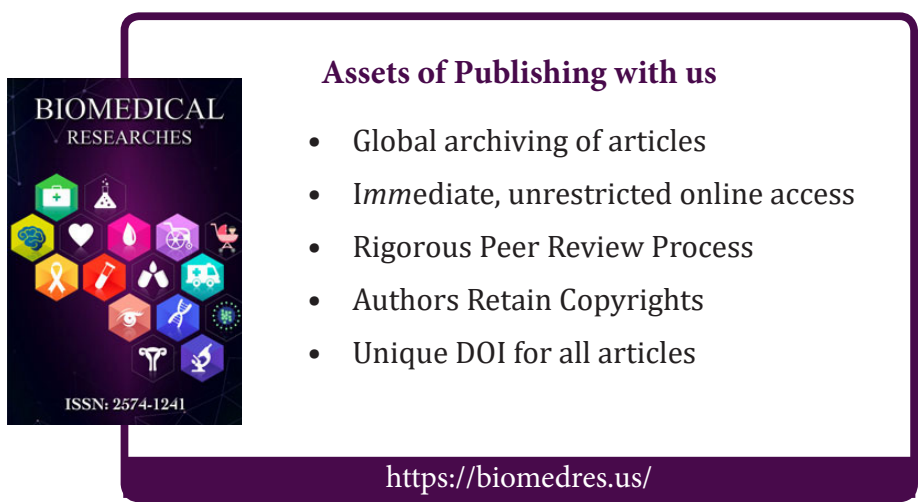

